\title{
THE ROLE OF HRCT IN EVALUATION OF AQUAIRED MIDDLE EAR CHOLESTEATOMA OTITIS PRIOR SURGERY
}

\author{
Ghada Abdulmonaem*, Rania almolla*,Ahmad Alsammak* \\ And Atef Hamed** \\ * Radiodiagnosis Department, Faculty of Medicine, Zagazig University, Egypt \\ **Otorhinolaryngology, Head and Neck Surgery Department, Faculty of Medicine, Zagazig University, \\ Egypt
}

\begin{abstract}
Objective: Otoscopic examination is the best method for diagnosis of cholesteatoma which treated by explorative surgery. The need for pre-operative imaging studies is controversial. This study assesses the accuracy and usefulness of a pre-operative high-resolution CT scan in depicting the status of the middle ear structures in the presence of cholesteatoma.

Patients and Methods: the surgical findings of 88 patients with acquired cholesteatoma were compared with the pre-operative CT findings in this prospective study. The following were analyzed: diagnostic features of cholesteatoma on CT, status of the middle ear structures (ossicles, facial nerve canal, bony labyrinth, tegmen tympani and scutum) and extension of the disease to the sinus tymapni and facial recess.

Results: eighty-three (94.3\%) cases had the two radiological features characteristic for cholesteatoma (a) a location typical for cholesteatoma in the epitympanum and mastoid antrum (b) bony erosion.

The radio-surgical agreement was excellent for the malleus (Kappa statistics, $\mathrm{K}=0.96$ ), stapes (0.91), bony labyrinth (0.94), tegmen tympani (0.82) and scutum (1), good for the incus (0.75), but poor for the facial nerve canal (0.39). The scan accurately predicted the extension of the disease to the sinus tympani and facial recess.

Conclusion: High-resolution CT scan is an important investigative tool prior to cholesteatoma surgery.There is good to excellent radio-surgical correlation in cholesteatoma for most middle ear structures except for the integrity of the facial canal. The scan alerts the surgeon to asymptomatic complication of the disease.
\end{abstract}

\section{INTRODUCTION}

$\mathrm{C}$ holesteatoma is an inflammatory disease of the temporal bone and generally develops in the middle ear[1]. It may be congenital or acquired. It is histologically composed of collection of keratinizing squamous epithelium in the middle ear cleft. It is accepted that most acquired cholesteatomas develop in a retraction pocket in the tympanic membrane, usually in the thinner pars flaccida, superiorly and extending towards the Prussak's space. Keratin squamous normally migrates from the tympanic membrane along the ear canal to the external auditory meatus[2].

Other mechanisms such asimplantation, migration through a perforation or even squamous metaplasia of middle ear mucosa, may be implicated [3]. The lesion is classically recognized by the presence of attic squamous on otoscopic examination[4].

Cholesteatoma is a potentially serious condition as it can progressively enlarge and erode into neighboring structures, giving rise to serious complications. Treatment of a suspected cholesteatoma is by surgical exploration and eradication of disease with atympano-mastoidectomyoperation[5].

The concept of surgery for middle ear cholesteatoma has traditionally been of exploration guided by awareness and anticipation of all, possibly asymptomatic complications[3].

Historically mastoid surgery has been undertaken with otoscopy, audiometry and possibly $\mathrm{x}$-rays as the only pre-operative investigations. Early computerized tomographic scanning had inherent limitations which resulted in poor spatial resolution and the inability to visualize fine bony details[6].Role played by preoperative imaging: evaluation of lesion extent (attic, antrum and mastoid); detection of complications, such as bone lysis and cerebromeningeal complications; and detection of anatomical variations (jugular dehiscence and lateralizedsinus).Special attention should be given to the tympanic sinus and to the facial recess[7]. 
In HRCT, the axial projection allows visualization of most of temporal bone anatomy, delineating the sinus tympani, facial recess, lateral semicircular canal, korner's septum, horizontal segment of the facial nerve canal, internal auditory canal, vestibular aqueduct, oval and round windows and temporal ear pathology [8].

Coronal scans is valuable in evaluating scutum, Prussak's space, roof of the tympanic cavity (tegmen), geniculate ganglion, oval window, stapes, ossicles, oval window, jugular fossa, internal auditory canal, vestibule and the vertical segment of the facial nerve canal.[8]

Axial images should include top of the petrous apex to the inferior tip of mastoid, and coronal images should be obtained from the anterior margin of petrous apex to the posterior margin of the mastoid [8].

Recently the advent of high-resolution CT scanning (HRCT) providing excellent spatial resolution has allowed super preoperative imaging of the fine anatomical details, evidence of the extent of the disease and a screen for asymptomatic complications[6]. HRCT is said to be capable of producing the fine details needed to detect lateral semicircular canal fistulae, exposed dura and facial canal dehiscence, and to demonstrate the ossicular chain[9].

The intent of this study was to evaluate the accuracy and the usefulness of high-resolution CT scan in depicting the status of the middle ear structures in patients undergoing surgery for cholesteatoma.

\section{PATIENTS AND METHODS}

This prospective study was carried out during the period from June 2014to March 2015, 88 patients (53 males, 35 females) with microscopically evident cholesteatoma who were listed for tympano-mastoid surgery underwent preoperative high resolution CT scanning of the petrous temporal bone.

The patient ages ranged from 14-57 years, (mean 31) and were referred from the ENT departments Zagazig University Hospitals. All patients were subjected to full clinical, audiologic, otoscopic and microscopic examination. Clinical data collected included information about hearing loss, otorrhea, otalgia, verigo and facial nerve function.

The HRCT scans were performed at the Radiology Departments Zagazig Hospitals using (General Eelectric Medical Systems, HiSpeed 6.03).

\section{HRCT examination technique:}

- HRCT was done to all patients with $512 \times 512$ matrix. Zooming and magnification were done for the petrous bone on each side. The HRCT scan protocol includes the following factors: $120 \mathrm{KV}, 200 \mathrm{MA}$, Scan time $1.5 \mathrm{sec}$,Slice thickness $1 \mathrm{~mm}$,interval $1 \mathrm{~mm}$,FOV 25mm,Mode: bone algorithm, ww 4000HU, WL 300HU, Collimation $1,5 \mathrm{~mm}$, and Pixel 0.25 $\mathrm{mm}^{2}$.

- Scanning was done in the direct axial and coronal planes for optimal demonstration of temporal bone structures.

\section{(a)Axial (horizontal projection):}

Scout view was obtained and sections were performed parallel to the anthropologic line(plane intersecting the inferior orbital rim and the superior margin of the external auditory canal).The sections were taken at $1 \mathrm{~mm}$ increments beginning at the level of the hypotympanum and jugular fossa and extending cephalically to the level of the arcuate eminence using line for localization.

\section{(b)Coronal(frontal)projection:}

With the neck fully extended, a lateral scout view was performed and sections performed nearly parallel to the ramus of mandible. The gantry of the scanner was tilted 15-20 to compensate for an incomplete extension of the neck. Sections were taken from the bony Eustachian tube anteriorly, extended posteriorly to the posterior wall of the mastoid bone. The coronal projection was not done in patients suffering from head trauma and obese patient with short neck. The coronal scanning also can be done with the patient supine with hyper extended neck.

HRCT images were interpreted in details to define:

(1)The type, location and extent of cholesteatoma.(2) Bony erosions of middle ear bony walls.(3) The 
integrity of the ossicularchain, facial nerve canal and labyrinth.(4) Involvement of hidden area, mastoid air cell system and condition.

\section{Contrast enhancement:}

- Non contrast HRCT scan of the temporal bone was done, unless there was suspected intracranial complications(in 17 cases). intravenous contrastwas administered in a dose of $1 \mathrm{ml} / \mathrm{kg}$ of iodinated contrast medium (low osmolar nonionic as omnipaque and teleprix).Pre and post contrast routine brain examination was done. Images were displayed using a soft tissue algorithm (WW 80-250, WL 35-55).

\section{Operative:}

Seventy-eight of our patients underwent canal wall down operation while the remaining 10 patients underwent canal wall up operation. Endoscope was used intraoperatively to assess hidden areas as sinus tympani and facial recess.

The operative findings were compared with the radiological findings.

Kappa statistics was used to measure the degree of agreement between surgical and radiological findings. Kappa values exceeding 0.75 represented excellent agreement, values between 0.4 and 0.75 fair to good agreement, and values less than 0.4 poor agreements.

\section{RESULTS}

Eighty-eight patients were included in our study. The majority of the patients $(80 \%)$ were older than 16 years of age. Sex distribution shows slight male predominance. $89 \%$ of patients had symptoms and signs for over 1 year prior to definitive surgery, and medical therapy had failed in most.

The lateral semicircular canal, tympanic portion of the facial nerve canal and extension of the disease to the sinus tympani and facial recess were better assessed using axial cans, while the tegmen tympani, vertical portion of the facial nerve canal, and scutum were more appreciated on the coronal scans. Both scans were complementary in evaluating the ossicular chain.

The HRCT scans showed the presence of non-dependent soft tissue mass in the epitympanum and mastoid antrum in all of our 88 (100\%) patients (table.1) Radiological evident of bone or ossicular erosion were present in $83(94.3 \%)$ cases and absent in 5 patients (Fig. 1). The two radiological features associated with cholesteatoma were present in $83(94.3 \%)$ cases.

The radio-surgical correlation of the middle ear structures is shown in table(2). The correlation was found to be excellent for the malleus, stapes, bony labyrinth, tegmen tympani, and scutum, good for the incus, but poor for the facial canal.

Table (1):HRCT and operative findings in 88 patients with cholesteatoma in our study:

\begin{tabular}{lllll}
\hline Affected bone & HRCT & \multicolumn{3}{c}{ No in surgery } \\
& No & \%o & \% \\
\hline Non-dependent soft tissue & 88 & 100 & 88 & 100 \\
\hline Scutum & 61 & 69 & 61 & 69 \\
\hline Incus & 66 & 75 & 63 & 71.6 \\
\hline Stapes & 27 & 30.7 & 24 & 27.3 \\
\hline Labryinth & 14 & 15.9 & 16 & 18.2 \\
\hline Tegmen & 16 & 18.2 & 17 & 19.3 \\
\hline Facial canal & 13 & 14.8 & 19 & 21.6 \\
\hline
\end{tabular}


Table (2):Operative findings and HRCT features correlation:

\begin{tabular}{|c|c|c|c|c|}
\hline & \multirow[t]{2}{*}{ Operative findings } & \multicolumn{3}{|c|}{ Radiological features } \\
\hline & & Intact & Abnormal* & Kappa \\
\hline \multirow[t]{2}{*}{ Scutum: } & Preserved & 27 & 0 & 1 \\
\hline & Blunted & 0 & 61 & \\
\hline \multirow[t]{2}{*}{ Incus: } & Preserved & 18 & 4 & 0.78 \\
\hline & Eroded & 3 & 63 & \\
\hline \multirow[t]{2}{*}{ Stapes: } & Preserved & 61 & 3 & 0.93 \\
\hline & Absent & 0 & 24 & \\
\hline \multirow[t]{2}{*}{ Labyrinth: } & Preserved & 72 & 0 & 0.93 \\
\hline & Fistula & 2 & 14 & \\
\hline \multirow[t]{2}{*}{ Tegmen: } & Preserved & 69 & 2 & 0.81 \\
\hline & Eroded & 3 & 14 & \\
\hline \multirow[t]{2}{*}{ Facial canal: } & Preserved & 64 & 5 & 0.4 \\
\hline & Dehiscent & 11 & 8 & \\
\hline
\end{tabular}

* Refers to bluntedscutum,destructedrnalus and incus, absence of stapes supra-structure, semicircular canal fistula, destructedtegmen, dehiscence of facial canal

\section{*Blunting of the Scutum (Fig. 3,4,5,6)}

There were 61 cases with surgically confirmed bluntedscutum all were detected by the HRCT. All 27 cases of preservedscutum were correctly predicted by the CT.

The scan predicted absolutely the extension of the pathology to the sinus tympani $(\mathrm{n}=34)$ and facial recess $(\mathrm{n}=17)$ (Fig.2,3).

\section{*State of the ossicles (Fig. 2,3 and 4)}

The incus was the most frequently eroded ossicle, followed by the malleus and the stapes. Out of the 66 incus which were found at surgery to be eroded, 63 were demonstrated with the scan. In the remaining 22 cases where the incus was preserved clinically the CT scan was incorrect in 4 cases. Error in assessment of the incus results from a failure in assessment of its long process.

All 34 eroded malleus were seen by the scan, however, two clinically intact malleoliwere misdiagnosed by the scan. All 24 cases of absent stapes were correctly predicted by the scan; however the scan failed to visualize an intact stapes in 3 cases.

\section{*Labyrinthine Fistula (Fig.6)}

There were 16 cases with surgically confirmed labyrinthine fistula, 14 involved the lateral semicircular canal, 14 of the 16 cases were visible on the scan, while the remaining two cases showed thinning of the lateral semicircular canal wall but with no discernible fistula on the scan. There were no false +ve radiographic interpretations.

\section{*Erosion of the Tegmen Tympani (Fig.5,6)}

Seventeen patients had erosion of the tegmen exposing the dura, the scan missed 3 cases of small dehiscence discovered at surgery. Of the remaining 71 cases, the scan misread two cases as having tegmen dehiscence when there was not any.

*Facial Canal Dehiscence (Fig.2)

Out of 19 cases with surgically confirmed facial -canal dehiscence, only 8 could be detected by the scan. In 5 cases there was CT evidence for a dehiscence in the tympanic segment of the canal, which was not detected intraoperative. The dehiscence involved the tympanic portion of the facial canal in all 19 cases. The vertical portion of the canal was not involved in any of our cases. 


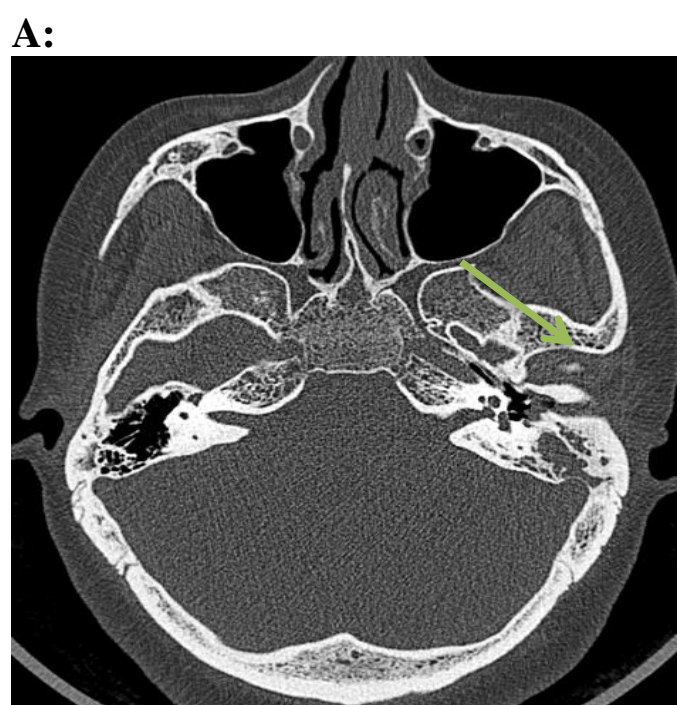

C:

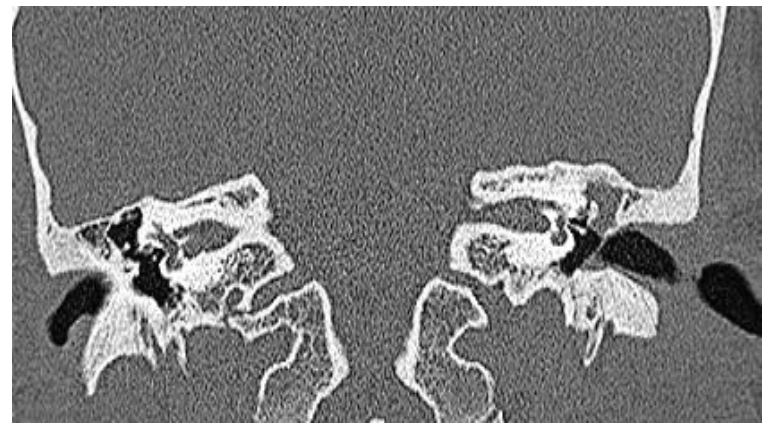

B:

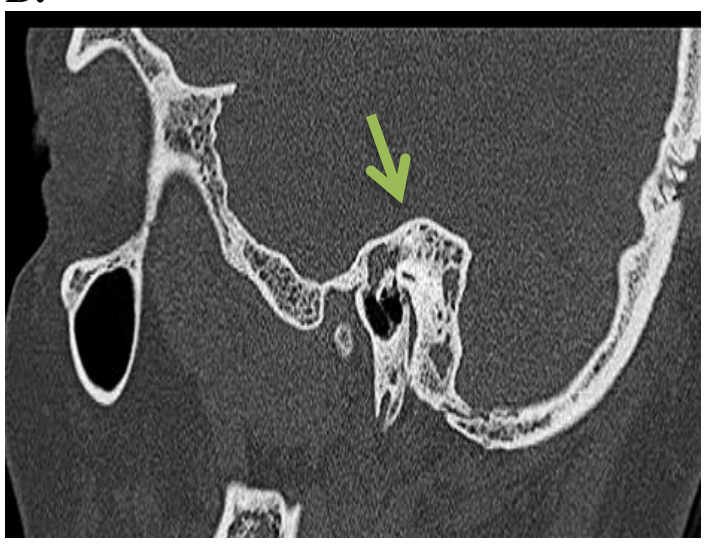

D:

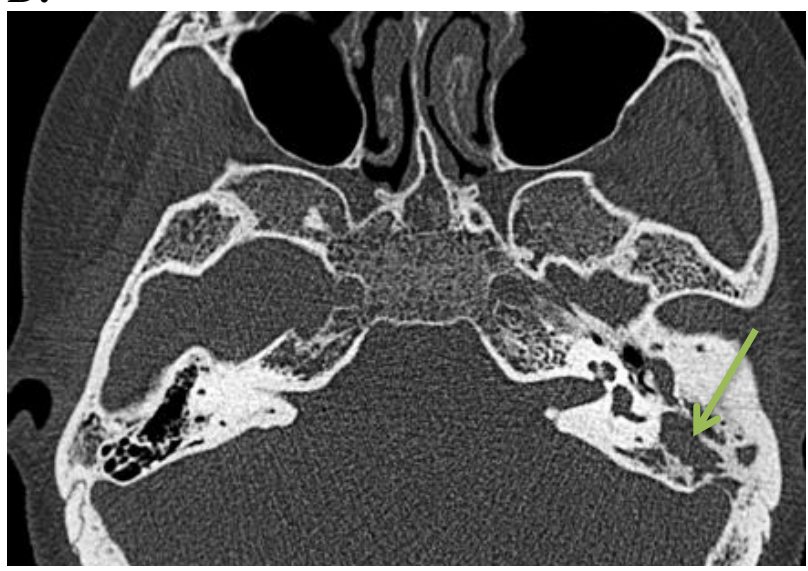

Fig. (1): Acase of left epitempaneum cholesteatoma (A.B,C) axial, sagittal and coronal images showing early erosion of the malleolus head and planting of the left scutum, intact handle and incus bone, D ) axial images shows extension of the soft tissue through the aditus to fill the mastoid air cells 
A:

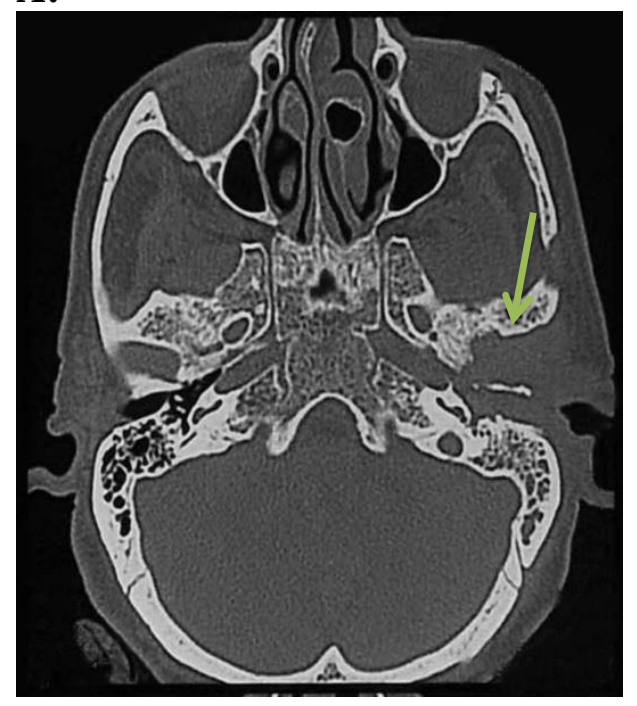

B:

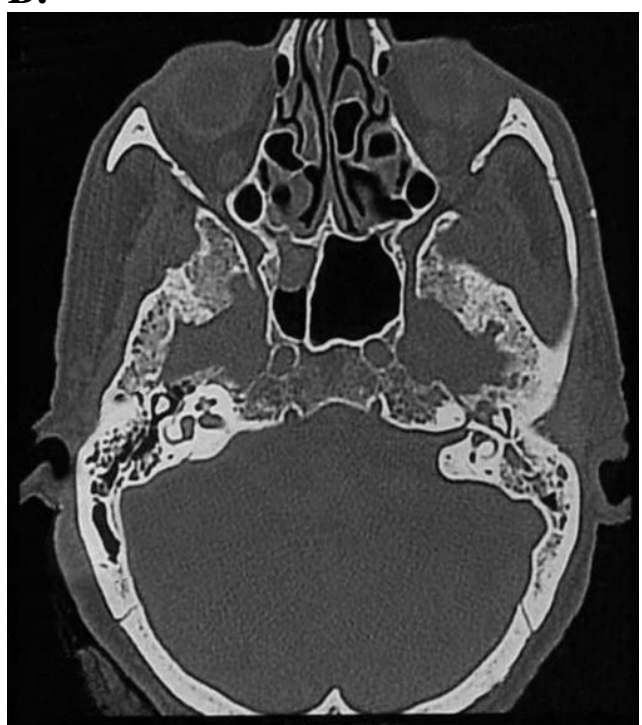

C:

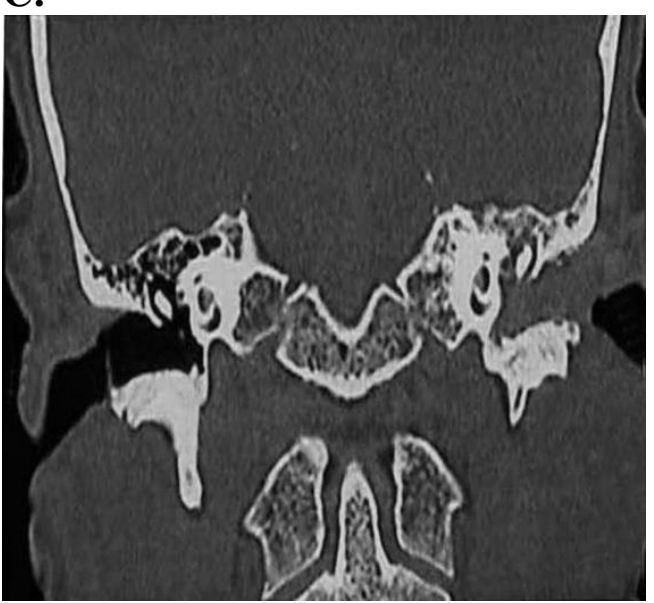

D:

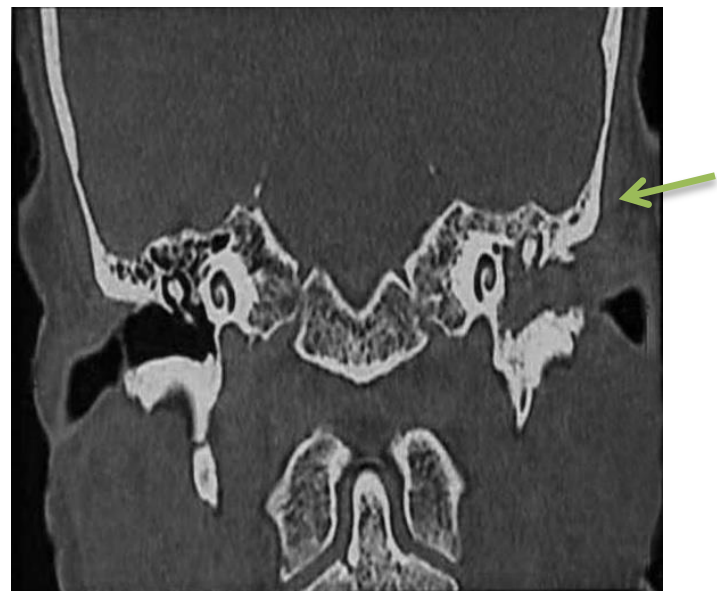

Fig. (2):Left side holotympanic cholesteatomawith erosion of tympanic segment of left facial nerve canal.Axial cuts Showingleft tympanic cavity is filled with non dependant soft tissue density mass extending to sinus tympani\&facial recess\& Eustachian tube, with opacification of mastoid air cells with resorption of left anterior inferior metal wall, however left head of malleus \&body of incus are still intact.(C\&D): Coronal cuts showing: Blunting of left scutum with erosion of tympanic segment of facial nerve canal, however, tegmen tympani is still intact. 

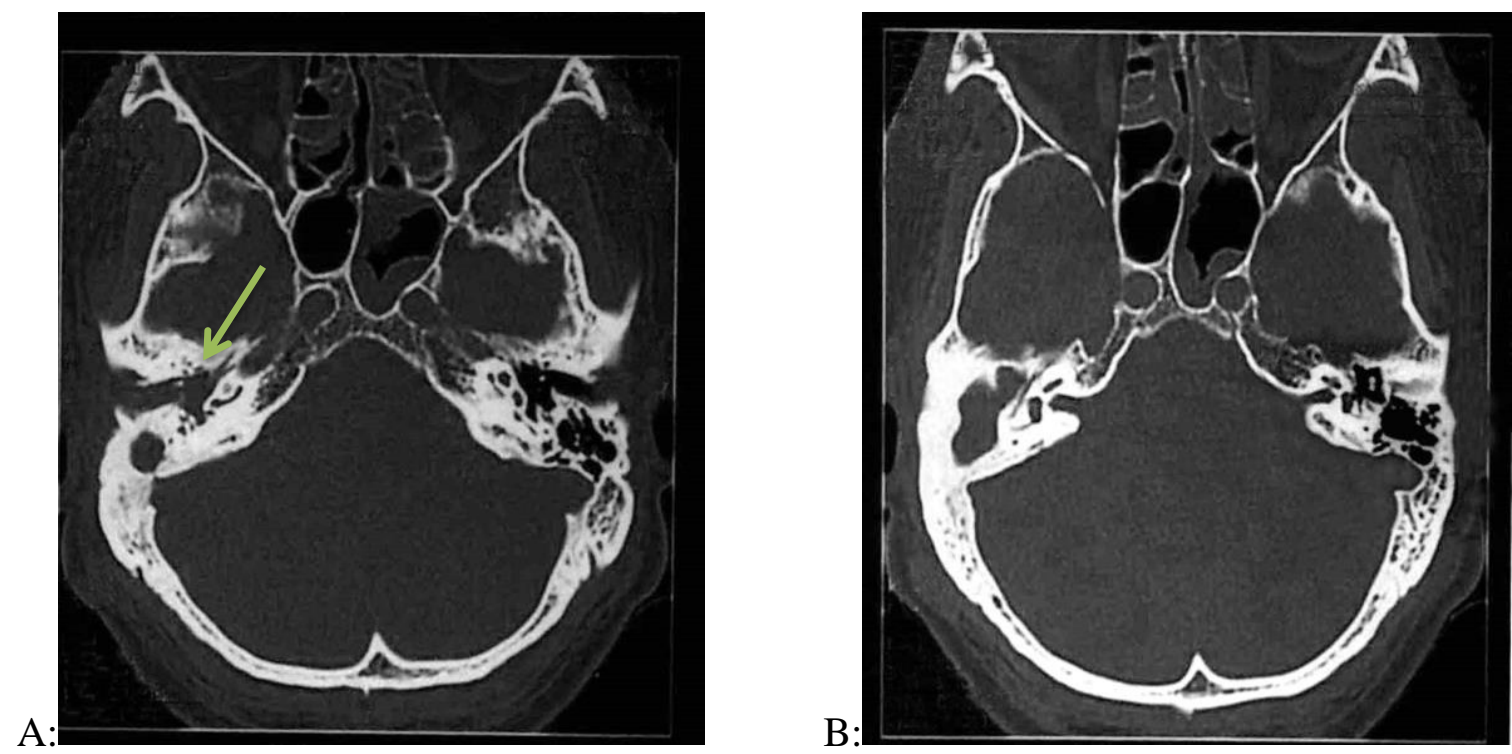

C:
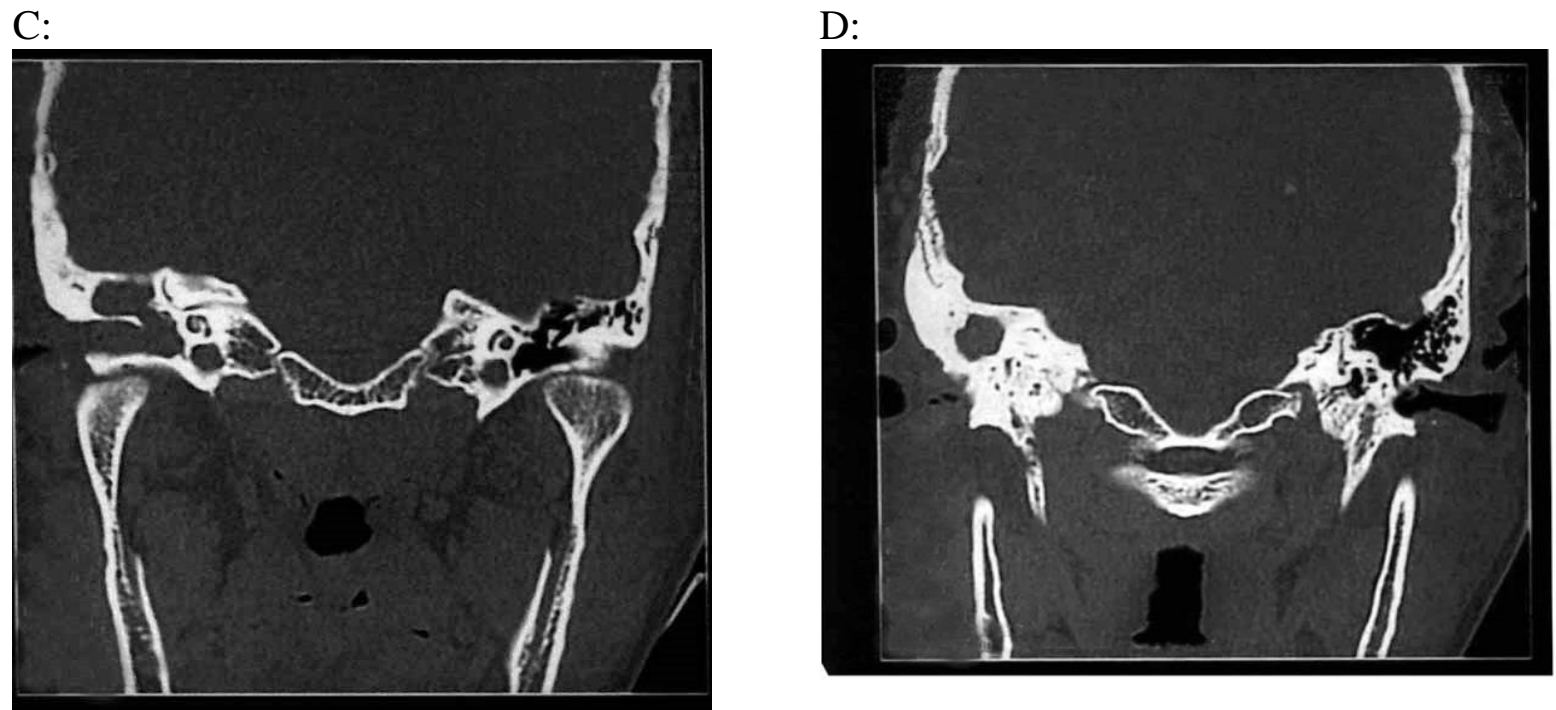

Fig. (3):Right holotympanic cholesteatomawith eroded ossicles.(A\& B): Axial cut showing:Right side non dependant soft tissue opacity filling mesotympanium extending to sinus tympani and facial recess as well as the right attic extending to mastoid antrum with widening of aditus ad antrum and complete erosion of the mastoid air cell (acellular mastoid) and erosion of the right ossicles. (C\& D): Coronal cut showing: complete erosion of ossicles and erosion of right scutum . 

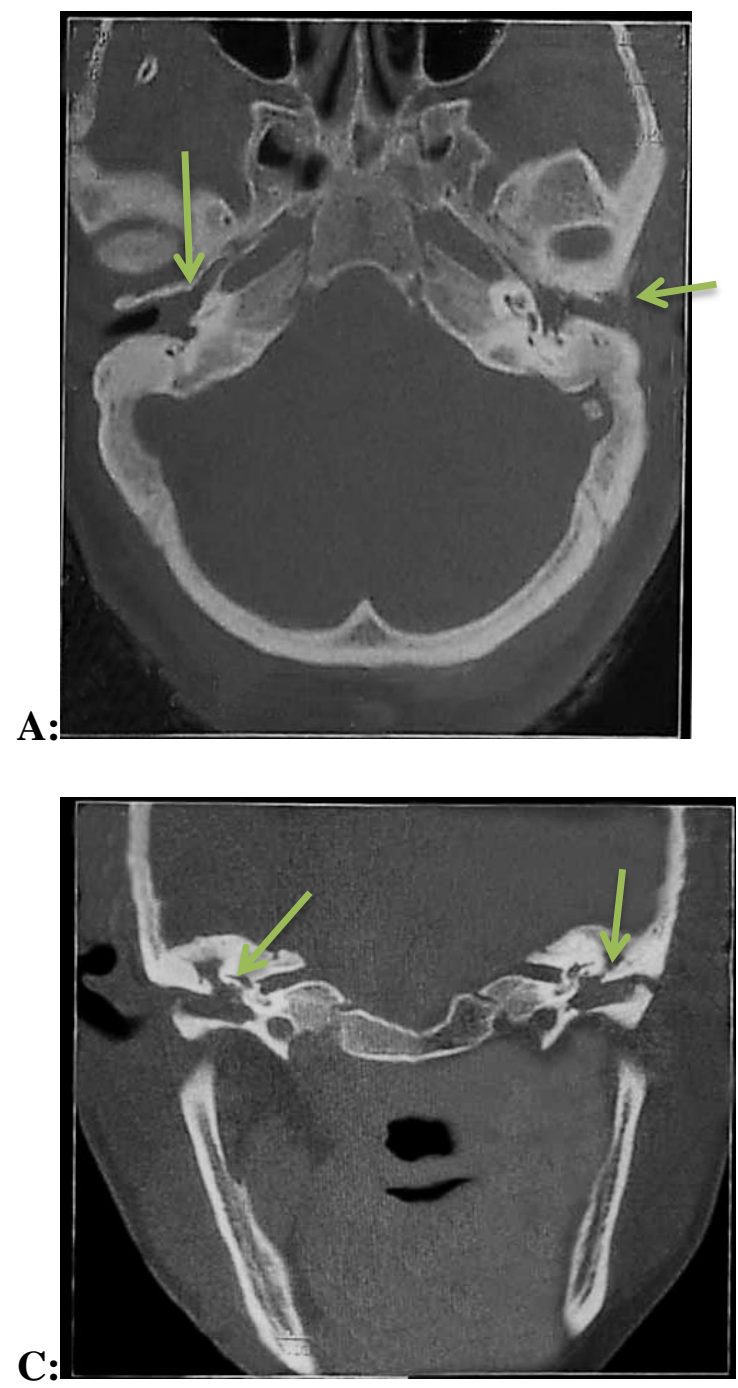

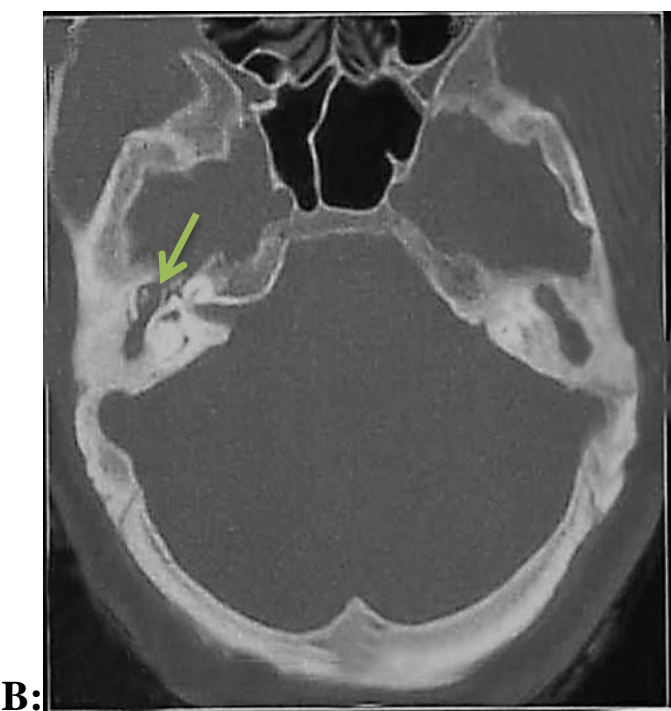

D:

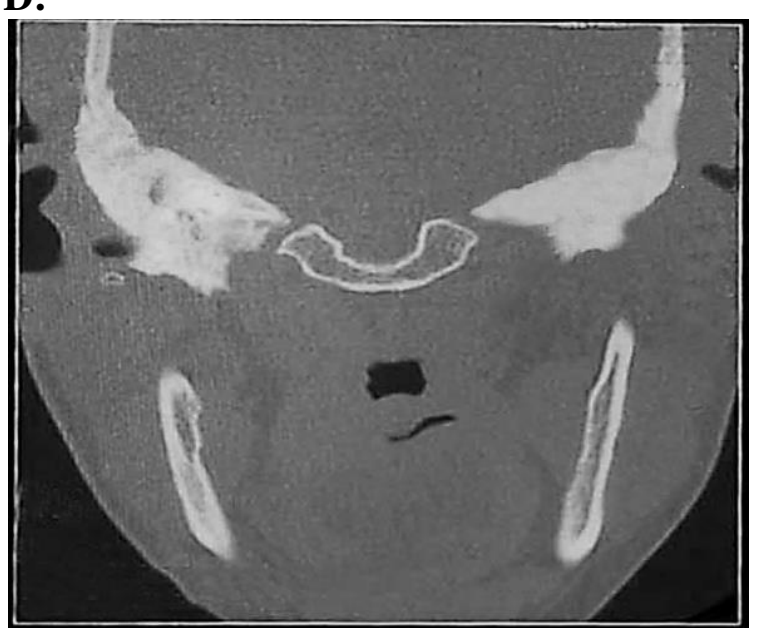

Fig. (4): Bilateral holotympanic cholesteatoma with bilateral eroded ossicles. (A\& B): Axial cut showing: Non dependant soft tissue opacity filling both middle ear cavities with involvement of sinus tympani \& facial recess, obliterating Eustachian tube on both sides. Filling attic and extending to matoidantrum with bilateral complete resorption of ossicles\& mastoid air cells (acellular mastoid). (C\& D): Coronal cut:Confirm on bilateralism of opacity filling the attic and mesotympanium with bilateral erosion of scutum \& ossicles. 

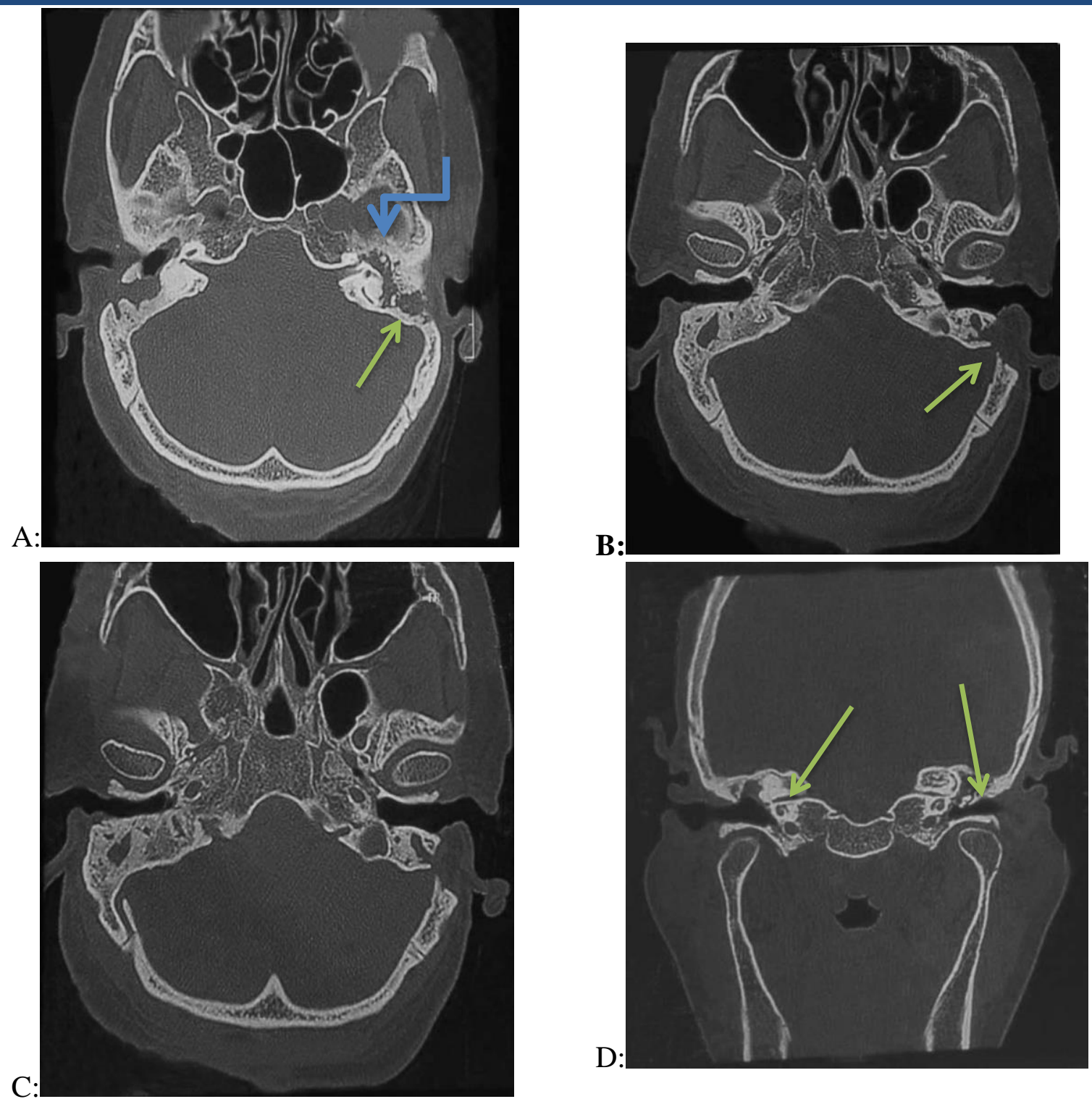

Fig. (5):Bilateral recurrent attico-antral cholesteatoma. Erosion of left sided sigmoid sinus plate. (A,B\& C):Axial cuts showing: Filling of epitympanium on both sides with independent soft tissue opacity extending to the mastoid antrum with absent ossicles on the right side,however remaining bone fragment on the left side. Notice erosion of left sided sigmoid sinus plate. (D): Coronal image showing: Erosion of scutum on right side and blunted on left side. 
A:

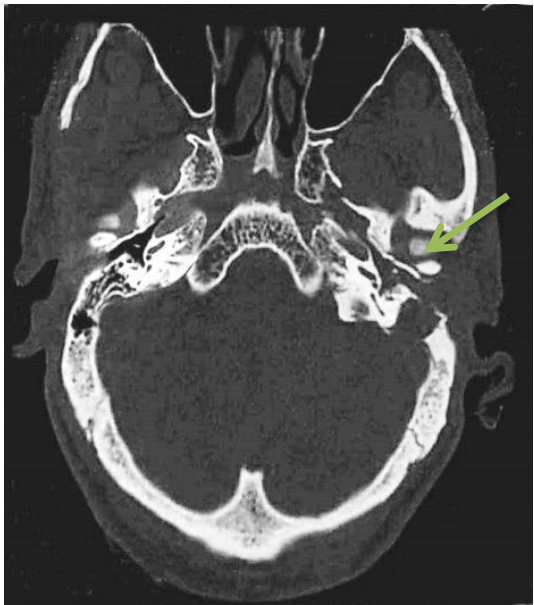

D:

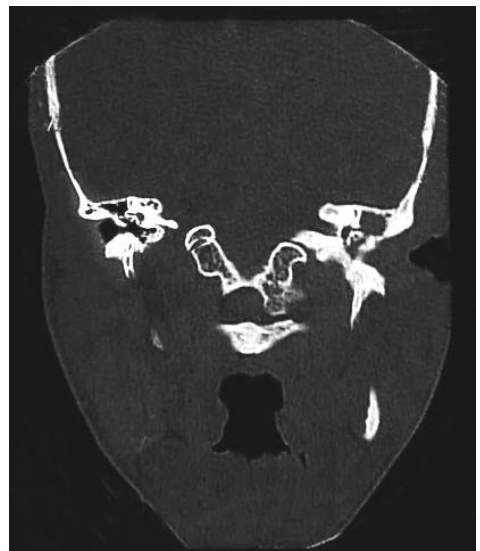

B:

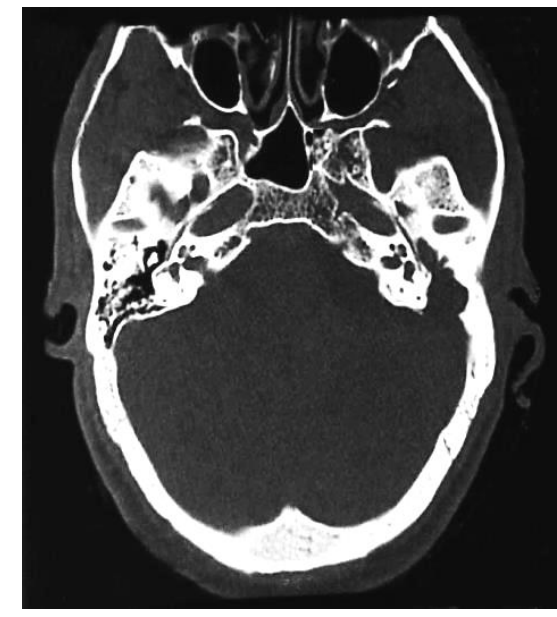

E:

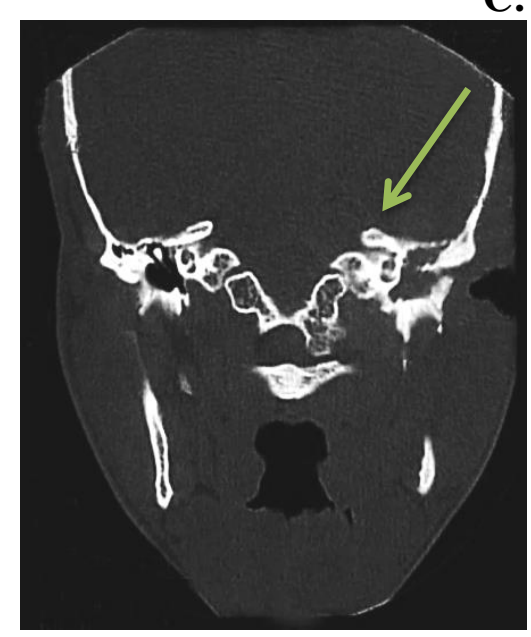

C:

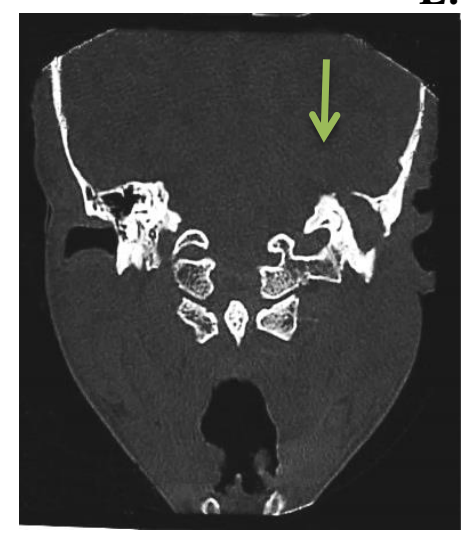

Fig6):Left extensive holotympanic cholesteatoma with erosion of ossicles sigmoid sinus plateandtegmen tympani with labyrinthine fistula. (A, B): Axial cut showing: Left side nondependent soft tissue opacity filling mesotympanium and attic extending to sinus tympani and facial recess, obliterating Eustachian tube with erosion of the left sigmoid sinus plate \&left lateral semicircular canal posterior metal wall as well as resorption of left ossicles mastoid air with erosion of scutum and ossicles on the coronal cuts there is Erosion of the left lateral semicircular canal.

\section{DISCUSSION}

High Resolution Computerized Tomography (HRCT) has significantly improved the contribution of imaging in the pre-operative assessment and management of Attico-antral type of chronic suppurative otitis media. In the presence of unsafe ear surgical treatment is the modality of treatment except in elderly and in patients with poor general medical condition.[10]

Cholesteatoma can be accurately diagnosed by the HRCT in the vast majority of cases by revealing a combination of a nondependent soft tissue mass in the epitympanum and antrum associated with bone erosion (4, 5,11). Relying on these criteria Mafee et al. [12]reported in his series of 48 patients with cholesteatoma that 46 of them $(96 \%)$ were diagnosed correctly with the pre-operative CT.
Chee and Tan[4]in their series of 36 patients with cholesteatoma found a nondependent mass in the epitympanum and antrum in $97 \%$ o of cases, bone erosion were reported in $94 \%$. These results are almost similar to that of our study as ossicles erosion was the mostly encountered complication seen in 66 patients (75\%).

The HRCT was $100 \%$ sensitive and $50 \%$ specific in diagnosing cholesteatoma. It is highly sensitive and specific in identifying the ossicular destruction.[13]

While prior knowledge of the state of the ossicles is probably not critical insofar as the operative risk is concerned, it has bearing on the likelihood of hearing preservation that can be achieved after surgery. For example, the hearing outcomes in patients with an intact 
stapes tend to be better than those where the stapes suprastructure is absent[14]. Pre-surgical knowledge of the status of the ossicular chain would allow the surgeon to be better advice the patient on the degree of hearing attainableafter surgery[4].

Our series showed excellent radio-surgical correlation for the malleus and stapes superstructures and good radio-surgical correlation for the incus. These results are in agreement with Chee and Tang who reported excellent radio-surgical correlation for the malleus and stapes $(\mathrm{K}=0.83$ and 0.94 respectively), but good correlation for the incus $(\mathrm{K}=0.62)$, Hassmann et al[15]also found good radio-surgical correlation in most middle ear structures except for the integrity of long process of incus.

Erosion of the long process of malleus was eroded in $45.7 \%$ of cases $(81.3 \%$ sensitivity and $94.1 \%$ specificity). Stapes superstructure could not be visualized in 11 cases $(31.4 \%)$ which were considered to be eroded ( $80 \%$ sensitivity and $95.7 \%$ specificity).[16]

On the other hand O'Reilly et al, [11] were able to correctly predict intact ossicular chain in only $50 \%$ of cases, the error in all cases being a failure to identify the long process of the incus and the stapes superstructures. Garber and Dort[17] showed only $40 \%$ sensitivity for detection of the stapes abnormalities.

Like many authors had experienced[11, 15]the error in assessment of the incus in our study was being a failure to probably assess its long process which according to Phelps and Wrightn[18]can be contributed to the partial volume averaging effect. However, Chintanetal[16] found that Erosion of the long process or body of incus was seen in $85.7 \%$ of cases (90\% sensitivity and $66.7 \%$ specificity)

Mafee et al[12]stated that cholesteatoma may occasionally erode the semicircular canals, particularly the lateral semicircular canal where it is exposed on the medial wall of the epitympanum. They reported complete radiosurgical agreement in detecting labyrinthine fistula. Colbertetal[19] showed excellent radiosurgical correlation $(K=0.76)$, this was also proved in our experience. Our study showed excellent radio-surgical correlation for the labyrinth fistula. We missed 2 cases of LSSC fistula, the scan showed thinning of the bone over the canal but no obvious fistulaisation. Careful dissection of the cholesteatoma matrix over the dome of the LSSC revealed a tiny bony canal fistula.[19]

On the contrary Jackler et al[20], favoring coronal scans, detected LSSC fistula in $50 \%$ of surgically confirmed cases, they also reported a high incidence of false positives. We agree with Garber and Dort[17] who reported that a normal scan in this region is unlikely to be followed by the discovery of pathology at the time of surgery.

We agree with many authors[2,6,8,11]who reported that the axial scan were the more satisfactory in detecting LSSC fistula because they depicted the canal in its entirety and were less likely to produce false results, but useful information could be also gained from coronal scans, yet they added that accurate assessment oil fistula requires both coronal and axial images.

The cochlea is easily identified but its cortex is rarely eroded[6], in our study we found only I case of cholesteatoma eroding the cochlea, erosion was confirmed surgically.(NO results for choclea)

The tegmen is visualized on coronal scans were it appears as a thin bony plate overlying the epitympanum and antrum[12]. We agree with Banergee et al[8]and Chee and Tan- who stated that tegmen erosion is well seen on coronal images but again misinterpretation may result from volume averaging effects. This was the case in one of our patients where the scan suggested the tegmen to be breached but surgically proven to be intact. The reverse is also possible whereby a dehiscent area may appear intact radiologically[4]. In 3 of our cases, small dehiscence discovered at surgery was not visualized by the preoperative scan.

Many authors[5,11,17,20]showed poor radiosurgical correlation for tegmen erosion, O'Reilly et al. [11] relied only on axial scan. Our results are better than that of Chintanet al [16], who found good radio-surgical correlation (93.8\% sensitivity and $88.2 \%$ specificity)andColbertet al [17], who also found good radio-surgical correlation $(\mathrm{K}=0.648)$

Facial canal dehiscence is a fairly common finding in suppurative ear diseases, usually occurring in the tympanic portion of the facial canal[21], the problem with partial 
volume averaging artifact is again evident here as the facial canal can be so thin, even in a nonpathological ear, as to appear dehiscent on a CT scan[4].

In our study we found difficulty in assessing the thin bone overlying the tympanic segment of facial canal, a problem also encountered by others $[4,8,11,17]$. Our radiosurgical agreement for facial canal dehiscence is poor $(\mathrm{K}=0.39)$ but comparable to those of Cheeand $\operatorname{Tan}[4](\mathrm{K}=0.30)$ and Banerjee et al.[8]who described the correlation to be unequivocal, Garber and Dort[17] detected about $40 \%$ of cases with facial canal dehiscence, O' Reilly et al[11] detected 4 out of 9 cases.

We agree with Walshe et al[22]who stated that scutum erosion was best visualized on coronal scans; in their series they detected 6 out of 7 eroded scutum. In our study CT clearly depicted all 38 cases of eroded scutum.

Besides giving information on the states of the middle ear structures, the CT scan also delineate the extent and location of the disease, cholesteatoma has tendency to reside in hidden areas as the sinus tympani and facial recess, those areas are difficult to assess even surgically, except with the use of intraoperative endoscope[4]. In our study we correctly assessed the extent of the disease in all cases, our results are in agreement with those ofWalshe et al[22].

We partially agree with Watts et al[6] who stated that axial scanning is only of value in demonstrating canal fistula and extension of the disease to the sinus tympani and facial recess, but is very insensitive to tegmen erosion, ossicular disease and facial nerve exposure, in our study axial scans was helpful in assessing the tympanic portion of the facial nerve and the ossicular chain.

Erosion of the LSSC by cholesteatoma in the absence of vertigo is presumably due to the cholesteatoma sealing the defect. If removed at mastoidectomy, such a patient may well become vertiginous. So Preoperative HRCT may have a medico-legal role before mastoid surgery. With evidence of erosion on the preoperative scan, any accusation of iatrogenic damage could be refuted[6].In our study we had 10 cases of asymptomatic lateral semicircular canal fistula detected by HRCT and confirmed at surgery)

While a definitive diagnosis of cholesteatoma can be made at the time of surgery, the scan picture may at time influence the decision and timing of surgical exploration. Scan evident of cholesteatoma with significant bony destruction or other complication could prompt the surgeon to operate earlier, particularly if polyps or a tortuous bony canal obscures visualization of the tympanic membrane and hinders clinical diagnosis[23].

High resolution computed tomography is today the best method of imaging to study chronic middle ear disease[15]. Mastoid surgery may be facilitated if imaging can reliably demonstrate the relevant anatomy, the nature and extent of the pathology and the presence of complications[6].

\section{CONCLUSION}

Approaching any middle ear pathology in the order of location and imaging characteristics would enable us in making a correct diagnosis in most of the cases. It is always important to make a note about the extent of the disease, and possible complications. This will serve as an efficient road mapfor the operating surgeon.

The advent of HRCT scan of the temporal bone has significantly enhanced the pre-operative evaluation of cholesteatoma. This study has shown that CT imaging for cholesteatoma accurately depicts the status of the middle ear structures, with the exception of the facial canal.

The scan delineates the location and extent of the disease and alerts the otologist to the presence of potential surgical hazards that may arise from the destructive nature of the disease.

HRCT is a valuable and useful investigative tool prior to cholesteatoma surgery.

\section{REFERENCES}

1. Sengupta A, Anwar T, Ghosh D, Basak B. A study of surgical management of chronic suppurative otitis media with cholesteatoma and its outcome. Indian J Otolaryngol Head Neck Surg 2010; 62:171-176.

2. Nemzek WR, Swartz JD. Temporal bone: inflammatory disease. In: Som PM, Curtin HD. Head and neck imaging. 4th ed. St Louis: Mosby; 2003. p.1184-99. 
3. Yates P, Flood LM, Banerjee A et al. CT scanning of cholesteatoma: what does the surgeon wants to know? Br J Radio] 75:1-8, 2002.

4. Chee NM, Tan TY. The value of preoperative high resolution CT scans in cholesteatoma surgery. Singapore Med J 2001, 42:155-159.

5. O'Donoghue GM, Bates GJ, Anslow P, et al. The predictive value of high resolution computed tomography in chronic suppurative ear disease. ClinOtolaryngol 12:89-96, 1987.

6. Watts S, Flood LM, Clifford K. A systemic approach to interpretation of computed tomography scans prior to surgery of middle ear cholesteatoma. J LaryngolOtol 114:248-253, 2000.

7. Ana FlviaAssis de vila, Bruna de Oliveira MelimAburjeli, Wanderval Moreira, Emlia Guerra Pinto Coelho Motta, Marcelo Almeida Ribeiro, Renata Lopes FurlettiCaldeira Diniz. Imaging evaluation of middle ear cholesteatoma: iconographic Essay. Radiol Bras. 2013 Jul/Ago;46(4):247-251

8. Banerjee A, Flood LM, Yaies $\mathrm{P}$ et al. Computed tomography in suppurative ear disease: does it influence management. J LaryngolOtol 117:454458,2003.

9. Razek AA, Huang BY. Lesions of the petrous apex: classification and findings at CT and MR imaging. Radiographics. 2012;32:151-73.

10. Meimaneijahromi

hoseinnejadarianiF,Arabkhani

A,

hoseeinnejadariani $\mathrm{Sh}$ Evaluation of cholesteatoma frequency in Patients with chronic otitis media. Iranian jornal of Otolaryngology, 2010;22(59):21-4.

11. O'reilly BJ, Chevretton EB, Wylie I et al. The value of CT scanning in chronic suppurative otitis media. J LaryngolOtol 105:990-994, 1991.

12. Mafee MF, Levin BC, ApplebaumEL, et al. Cholesteatoma of the middle ear and mastoid. OtolayngolClin North Am 21:265293, 1988.
13. Abhijeet Kumar Sinha, Sheikh Nizamuddin Mustafa, Ehtesham Ahmad Raushan, Gireesh Kumar. Role of High Resolution Computed Tomography in Cholesteatoma. International Journal of Scientific Study, Vol 2, Issue 8, 2014

14. Cook JA, Krishman S, Fagan PA. Hearing results following modified radical versus canal up mastoidectomy. Ann Otol Rhino] Laryngol 105 (5):379-383, 1996.

15. Hassmann E, Goscik E, Olenski J, et al. Computerized tomography in pre-operative imaging of middle ear cholesteatoma: Otolaryngol Pol. (Abstract) 57(2):243-249, 2003.

16. Chintan Shah, Pinak Shah and Satish Shah. Role of HRCT temporal bone in preoperative evaluation of cholesteatoma. international journal of medical science and public health, vol 3 , issue $1 ; 69: 72,2014$

17. Garber LZ, Dort JC. Cholesteatoma. Diagnosis and staging by CT scan. J Otolaryngol 23(2):121124, 1994.

18. Phelps PD, WrightA. Imaging cholesteatoma. ClinRadiol 41:156-162, 1990.

19. Colbert K, Gupta V and M. Ravisankar. Computer tomography and surgical correlation in unsafe ear. IOSR Journal of Dental and Medical Sciences, Volume 13, Issue 6 Ver. IV ( 2014), PP 07-12

20. Jakler RK, Dillon WP, Schindler RA. Computed tomography in suppurative ear disease: a correlation of surgical and radiographic findings. Laryngoscope 94:746-752, 1984

21. Fuse $T$, Tada $Y$, Aoyagi $M$, et al. CT detection of facial canal dehiscence and semicircular canal fistula: Comparison with surgical findings..JComput Ass. Tomography 20(2):221-224, 1996.

22. Walshe P, Mcconn W, Brennan P et al. The role of computerized tomography in the preoperative assessment of chronic suppurative otitis media.ClinOtolaryngol 27:95-97, 2002.

23. Belvins NH, Carter BL. Routine preoperative imaging in chronic ear surgery. Am J Otol 19: 527-538, 1998. 